\title{
Existence of multiple solutions for nonlinear multi-point boundary value problems
}

\author{
Hongyu Li ${ }^{i^{*}}$, Yang Chen ${ }^{1}$ and Junting Zhang ${ }^{1}$
}

\section{"Correspondence:} sdlhy1978@163.com

${ }^{1}$ College of Mathematics and Systems Science, Shandong

University of Science and

Technology, Qingdao, P.R. China

\begin{abstract}
In this paper, we study some nonlinear second order multi-point boundary value problems. We first give a lemma about the characteristic values of the corresponding linear operator. Then, by fixed point theorems in the recent existing literature, we obtain the existence of multiple solutions for these nonlinear second order multi-point boundary value problems, including two positive solutions, two negative solutions, and one sign-changing solution.
\end{abstract}

MSC: 34B15; 34B18; 47H11

Keywords: Fixed point theorem; Characteristic value; Sign-changing solution; Multi-point boundary value problem

\section{Introduction}

In this paper, we study the following nonlinear multi-point boundary value problem:

$$
\left\{\begin{array}{l}
-u^{\prime \prime}(t)=g(t, u(t)), \quad 0 \leq t \leq 1, \\
u^{\prime}(0)=0, \quad u(1)=\sum_{i=1}^{m-2} \alpha_{i} u\left(\beta_{i}\right),
\end{array}\right.
$$

where $g:[0,1] \times(-\infty,+\infty) \rightarrow(-\infty,+\infty)$ is continuous; $0<\beta_{1}<\beta_{2}<\cdots<\beta_{m-2}<1 ; \alpha_{i}>$ $0(i=1,2, \ldots, m-2)$ with $\sum_{i=1}^{m-2} \alpha_{i}<1$.

The multi-point boundary value problem is an important part of boundary value problems for ordinary differential equations, which arise in physics and applied mathematics (see [1]). In 1992, Gupta considered nonlinear three-point boundary value problems (see [2]). Since then, many authors have considered the existence of nontrivial solutions for nonlinear multi-point boundary value problems and obtained many great results. We can refer to [3-18] and the references therein. For example, in [4], Xu has studied the following multi-point boundary value problem:

$$
\left\{\begin{array}{l}
-x^{\prime \prime}(t)=f(x), \quad t \in[0,1], \\
x(0)=0, \quad x(1)=\sum_{i=1}^{m-2} \alpha_{i} x\left(\eta_{i}\right),
\end{array}\right.
$$

where $f:(-\infty,+\infty) \rightarrow(-\infty,+\infty)$ is continuous; $0<\eta_{1}<\eta_{2}<\cdots<\eta_{m-2}<1 ; \alpha_{i}>0(i=$ $1,2, \ldots, m-2)$. In [4], the author gave the result about the characteristic values of the

(c) The Author(s) 2020. This article is licensed under a Creative Commons Attribution 4.0 International License, which permits use, sharing, adaptation, distribution and reproduction in any medium or format, as long as you give appropriate credit to the original author(s) and the source, provide a link to the Creative Commons licence, and indicate if changes were made. The images or other third party material in this article are included in the article's Creative Commons licence, unless indicated otherwise in a credit line to the material. If material is not included in the article's Creative Commons licence and your intended use is not permitted by statutory regulation or exceeds the permitted use, you will need to obtain permission directly from the copyright holder. To view a copy of this licence, visit http://creativecommons.org/licenses/by/4.0/. 
relevant linear operator. In addition, the author obtained the existence of multiple positive solutions and sign-changing solutions for BVP (1.2) by applying Leray-Schauder degree methods.

In [5], Zhang and Sun studied the following nonlinear multi-point boundary value problem:

$$
\left\{\begin{array}{l}
-(L y)(t)=h(t) f(y(t)), \quad t \in(0,1), \\
y^{\prime}(0)=0, \quad y(1)=\sum_{i=1}^{m-2} a_{i} y\left(\xi_{i}\right),
\end{array}\right.
$$

where $(L y)(t)=\left(p(t) y^{\prime}(t)\right)^{\prime}+q(t) y(t), f:[0,+\infty) \rightarrow[0,+\infty)$ is continuous; $h:(0,1) \rightarrow$ $[0,+\infty)$ is continuous, and $h$ is singular at $t=0$ and $t=1 ; 0<\xi_{1}<\xi_{2}<\cdots<\xi_{m-2}<1$; $a_{i}>0(i=1,2, \ldots, m-2)$. The authors proved the existence of the first eigenvalue of the relevant linear operator, and they considered the existence of positive solutions for BVP (1.3). The method they used is the fixed point index theory.

In [15], Li considered the following second order three-point boundary value problem:

$$
\left\{\begin{array}{l}
-y^{\prime \prime}(t)=f(t, u(t)), \quad t \in[0,1], \\
y^{\prime}(0)=0, \quad y(1)=\alpha y(\eta),
\end{array}\right.
$$

where $\eta \in(0,1), \alpha \in(0,1) ; f:[0,1] \times(-\infty,+\infty) \rightarrow(-\infty,+\infty)$ is continuous. The author also studied the characteristic values of the relevant linear operator about BVP (1.4). By means of fixed point theorems with lattice structure, the author has obtained the existence results of negative and sign-changing solution for BVP (1.4) for superlinear case.

Inspired by [3-18], we consider boundary value problem (1.1) in this paper. By the existing fixed point theorems due to Sang et al. [19], we obtain the existence results of multiple nontrivial solutions for BVP (1.1) for asymptotically linear case, including two positive solutions, one sign-changing, and two negative solutions. Characteristic value is an important index of the linear operator. One of the features of this paper is that we give a lemma about the characteristic values of the relevant linear operator about BVP (1.1). The other feature of this paper is that the obtained main theorems are new results for BVP (1.1), which improve and generalize BVP (1.4).

The rest of the paper is organized as follows. We introduce the definition of e-continuous operator and the used fixed point theorems due to Sang et al. [19] in Sect. 2. We give the main lemma about the characteristic values of the relevant linear operator, prove some auxiliary lemmas that we need, and obtain the main result of the existence of multiple solutions for BVP (1.1) in Sect. 3. We provide an example to illustrate our main result in Sect. 4.

\section{Preliminaries}

In the following, we mainly introduce the e-continuous operator and list the used fixed point theorems due to Sang et al. [19]. For detailed concepts and properties about the cone, we can refer to [21-23].

Let $E$ be a Banach space, $P$ be a cone of $E$. Let $A$ be an operator. If $A u=u$ with $u \notin$ $P \cup(-P)$, then $u$ is said to be a sign-changing fixed point of $A$. The linear operator $B$ is said to be positive if $B(P) \subset P$. 
Definition 2.1 (see [20]) Let $A: D \rightarrow E$ be an operator, $e \in P \backslash\{\theta\}$, and $u_{0} \in D$. If, for any given $\epsilon>0$, there exists $\delta>0$ such that

$$
-\epsilon e \leq A u-A u_{0} \leq \epsilon e, \quad \text { for all } u \in D \text { with }\left\|u-u_{0}\right\|<\delta,
$$

then $A$ is said to be e-continuous at $u_{0}$. If $A$ is e-continuous at every point $u \in D$, then $A$ is said to be e-continuous on $D$.

Lemma 2.1 (see [19]) Let $E$ be a Banach space, $P$ be a normal and total cone of $E ; B$ : $E \rightarrow E$ be a positive linear completely continuous operator and be also e-continuous on $E$; $F: E \rightarrow E$ be a continuous and bounded increasing operator and $A=B F$. Assume that

(i) there exist $x_{1} \in P \backslash\{\theta\}$ and $x_{2} \in(-P) \backslash\{\theta\}$ such that $A x_{1} \leq x_{1}, x_{2} \leq A x_{2}$; and there exists $\alpha>0$ such that $\alpha e \leq x_{1}, x_{2} \leq-\alpha e$;

(ii) $F(\theta)=\theta$, $F$ is Fréchet differentiable at $\theta$; and $A_{\theta}^{\prime}$ has a characteristic value $\lambda_{0}<1$ with a characteristic function $\psi$ satisfying $\mu_{1} e \leq \psi \leq \mu_{2} e$, where $\mu_{1}>0, \mu_{2}>0$;

(iii) the Fréchet derivative $A_{\infty}^{\prime}$ at $\infty$ exists; $A_{\infty}^{\prime}$ is increasing; $r\left(A_{\infty}^{\prime}\right)>1 ; 1$ is not a a characteristic value of $A_{\infty}^{\prime}$.

Then $A$ has at least two positive fixed points, one sign-changing solution, fixed point, and two negative fixed points.

\section{Main results}

Let $E=C[0,1]$. Define the norm $\|u\|=\max _{t \in[0,1]}|u(t)|$ on $E$, then $E$ is a Banach space. Let $P=\{u \in E \mid u(t) \geq 0, t \in[0,1]\}$. It is obvious that $P$ is a normal and total cone of $E$ (see [21-23]).

For convenience, we first give the following assumptions to be used in the rest of this paper.

$\left(\mathrm{H}_{1}\right)$ The sequence of positive solutions of the equation

$$
\cos \sqrt{x}=\sum_{i=1}^{m-2} \alpha_{i} \cos \left(\beta_{i} \sqrt{x}\right)
$$

is $\lambda_{1}<\lambda_{2}<\cdots<\lambda_{n}<\cdots$.

$\left(\mathrm{H}_{2}\right) g(t, 0)=0$ and $g(t, x)$ is increasing in $x$ uniformly on $t \in[0,1]$.

$\left(\mathrm{H}_{3}\right) \lim _{x \rightarrow 0} \frac{g(t, x)}{x}=\eta, \forall t \in[0,1]$ and $\eta>\lambda_{1}$, where $\lambda_{1}$ is defined by $\left(\mathrm{H}_{1}\right)$.

$\left(\mathrm{H}_{4}\right) \lim _{x \rightarrow \infty} \frac{g(t, x)}{x}=\gamma, \forall t \in[0,1]$ and $\gamma>\lambda_{1}, \gamma \neq \lambda_{k}$, where $\lambda_{k}$ is defined by $\left(\mathrm{H}_{1}\right), k=$ $2,3, \ldots$

$\left(\mathrm{H}_{5}\right)$ There exist $\tau>0, w>0$, and $C>0$ such that

$$
\frac{g(t, \tau)}{\tau}<C, \quad \frac{g(t,-w)}{-w}<C \quad \text { for any } t \in[0,1]
$$

where $0<C<\left(1-\sum_{i=1}^{m-2} \alpha_{i}\right)$.

Lemma 3.1 (see [5]) For $y(t) \in E$, the following boundary value problem

$$
\left\{\begin{array}{l}
u^{\prime \prime}(t)+y(t)=0, \quad t \in[0,1], \\
u^{\prime}(0)=0, \quad u(1)=\sum_{i=1}^{m-2} \alpha_{i} u\left(\beta_{i}\right),
\end{array}\right.
$$


has a unique solution

$$
u(t)=\int_{0}^{1} G(t, s) y(s) d s, \quad 0 \leq t \leq 1
$$

where

$$
\begin{aligned}
G(t, s) & =k(t, s)+\sum_{i=1}^{m-2} \widetilde{k}_{i}(t, s)+(1-s)\left(\sum_{i=1}^{m-2} \alpha_{i}\right)\left(1-\sum_{i=1}^{m-2} \alpha_{i}\right)^{-1}, \quad t, s \in[0,1] . \\
k(t, s) & = \begin{cases}1-s, & 0 \leq t \leq s \leq 1, \\
1-t, & 0 \leq s \leq t \leq 1,\end{cases} \\
\tilde{k}_{i}(t, s) & = \begin{cases}-\alpha_{i}\left(1-\sum_{i=1}^{m-2} \alpha_{i}\right)^{-1}\left(\beta_{i}-s\right), & 0 \leq s \leq \beta_{i}, t \in[0,1], \\
0, & \beta_{i} \leq s \leq 1, t \in[0,1],\end{cases} \\
i=1,2, \ldots, m-2 . &
\end{aligned}
$$

Define the following operators:

$$
\begin{aligned}
& (T u)(t)=\int_{0}^{1} G(t, s) g(s, u(s)) d s, \\
& (L u)(t)=\int_{0}^{1} G(t, s) u(s) d s, \\
& (G u)(t)=g(t, u(t)),
\end{aligned}
$$

where $T=L G, G(t, s)$ is defined by (3.2).

Obviously, $T: E \rightarrow E$ is completely continuous (see [5]).

Lemma 3.2 Assume that $\left(\mathrm{H}_{1}\right)$ holds. Then the sequence of positive characteristic values of the linear operator $L$ defined by (3.6) is

$$
\lambda_{1}<\lambda_{2}<\cdots<\lambda_{n}<\cdots
$$

and the positive characteristic values $\lambda_{n}$ have algebraic multiplicity one.

Proof Let $\xi$ be a positive characteristic value and $u(t)$ be a characteristic function corresponding to the characteristic value $\xi$.

From Lemma 3.1, we obtain

$$
\begin{aligned}
& u^{\prime \prime}(t)+\xi u(t)=0, \quad 0 \leq t \leq 1 \\
& u^{\prime}(0)=0, \quad u(1)=\sum_{i=1}^{m-2} \alpha_{i} u\left(\beta_{i}\right) .
\end{aligned}
$$


Then the form of the general solution for the differential equation (3.8) is

$$
u(t)=C_{1} \sin (t \sqrt{\xi})+C_{2} \cos (t \sqrt{\xi}), \quad \forall t \in[0,1] .
$$

Since $u^{\prime}(0)=0$, we know that $C_{1}=0$. Then (3.9) can be reduced to

$$
u(t)=C_{2} \cos (t \sqrt{\xi}), \quad \forall t \in[0,1] .
$$

From $u(1)=\sum_{i=1}^{m-2} \alpha_{i} u\left(\beta_{i}\right)$, we have

$$
\cos \sqrt{\xi}=\sum_{i=1}^{m-2} \alpha_{i} \cos \left(\beta_{i} \sqrt{\xi}\right) .
$$

By $\left(\mathrm{H}_{1}\right)$, we know that $\xi$ is one of the values $\lambda_{1}<\lambda_{2}<\cdots<\lambda_{n}<\cdots$, and the corresponding characteristic function is

$$
u_{n}(t)=C \cos \left(t \sqrt{\lambda_{n}}\right), \quad t \in[0,1]
$$

where $C$ is a nonzero constant.

By ordinary method, we can know that

$$
\operatorname{dim} \operatorname{Ker}\left(I-\lambda_{n} L\right)=1
$$

We need to prove that

$$
\operatorname{Ker}\left(I-\lambda_{n} L\right)=\operatorname{Ker}\left(I-\lambda_{n} L\right)^{2} .
$$

It is obvious that we only need to prove that

$$
\operatorname{Ker}\left(I-\lambda_{n} L\right)^{2} \subset \operatorname{Ker}\left(I-\lambda_{n} L\right)
$$

Take any $u \in \operatorname{Ker}\left(I-\lambda_{n} L\right)^{2}$. If $\left(I-\lambda_{n} L\right) u \neq \theta$, then $\left(I-\lambda_{n} L\right) u$ is a characteristic function of the linear operator $L$ corresponding to the characteristic value $\lambda_{n}$. So we have

$$
\left(I-\lambda_{n} L\right) u=b \cos \left(t \sqrt{\lambda_{n}}\right), \quad t \in[0,1],
$$

where $b$ is a nonzero constant.

Hence we easily obtain that

$$
\begin{aligned}
& u^{\prime \prime}(t)+\lambda_{n} u(t)=-\lambda_{n} b \cos \left(t \sqrt{\lambda_{n}}\right), \quad 0 \leq t \leq 1 . \\
& u^{\prime}(0)=0, \quad u(1)=\sum_{i=1}^{m-2} \alpha_{i} u\left(\beta_{i}\right) .
\end{aligned}
$$

Then the form of the general solution for the differential equation (3.13) is

$$
u(t)=C_{1} \sin \left(t \sqrt{\lambda_{n}}\right)+C_{2} \cos \left(t \sqrt{\lambda_{n}}\right)-\frac{b}{4} \cos \left(2 t \sqrt{\lambda_{n}}\right) \cos \left(t \sqrt{\lambda_{n}}\right)
$$




$$
-\left(\frac{b \sin \left(2 t \sqrt{\lambda_{n}}\right)}{4}+\frac{b t \sqrt{\lambda_{n}}}{2}\right) \sin \left(t \sqrt{\lambda_{n}}\right), \quad t \in[0,1] .
$$

Since $u^{\prime}(0)=0$, we know that $C_{1}=0$. By (3.14) and $\cos \sqrt{\lambda_{n}}=\sum_{i=1}^{m-2} \alpha_{i} \cos \left(\beta_{i} \sqrt{\lambda_{n}}\right)$, we have

$$
\begin{aligned}
u(1)= & C_{2} \cos \sqrt{\lambda_{n}}-\frac{b}{4} \cos \left(2 \sqrt{\lambda_{n}}\right) \cos \sqrt{\lambda_{n}}-\left(\frac{b \sin \left(2 \sqrt{\lambda_{n}}\right)}{4}+\frac{b \sqrt{\lambda_{n}}}{2}\right) \sin \sqrt{\lambda_{n}} \\
= & C_{2} \sum_{i=1}^{m-2} \alpha_{i} \cos \left(\beta_{i} \sqrt{\lambda_{n}}\right)-\frac{b}{4}\left(1-2 \sin ^{2} \sqrt{\lambda_{n}}\right) \sum_{i=1}^{m-2} \alpha_{i} \cos \left(\beta_{i} \sqrt{\lambda_{n}}\right) \\
& -\frac{b}{2} \sin ^{2} \sqrt{\lambda_{n}} \sum_{i=1}^{m-2} \alpha_{i} \cos \left(\beta_{i} \sqrt{\lambda_{n}}\right)-\frac{b \sqrt{\lambda_{n}}}{2} \sin \sqrt{\lambda_{n}} \\
= & C_{2} \sum_{i=1}^{m-2} \alpha_{i} \cos \left(\beta_{i} \sqrt{\lambda_{n}}\right)-\frac{b}{4} \sum_{i=1}^{m-2} \alpha_{i} \cos \left(\beta_{i} \sqrt{\lambda_{n}}\right)-\frac{b \sqrt{\lambda_{n}}}{2} \sin \sqrt{\lambda_{n}}
\end{aligned}
$$

and

$$
\begin{aligned}
\sum_{i=1}^{m-2} \alpha_{i} u\left(\beta_{i}\right)= & C_{2} \sum_{i=1}^{m-2} \alpha_{i} \cos \left(\beta_{i} \sqrt{\lambda_{n}}\right)-\frac{b}{4} \sum_{i=1}^{m-2} \alpha_{i} \cos \left(2 \beta_{i} \sqrt{\lambda_{n}}\right) \cos \left(\beta_{i} \sqrt{\lambda_{n}}\right) \\
& -\frac{b}{4} \sum_{i=1}^{m-2} \alpha_{i} \sin \left(2 \beta_{i} \sqrt{\lambda_{n}}\right) \sin \left(\beta_{i} \sqrt{\lambda_{n}}\right)-\frac{b \sqrt{\lambda_{n}}}{2} \sum_{i=1}^{m-2} \alpha_{i} \beta_{i} \sin \left(\beta_{i} \sqrt{\lambda_{n}}\right) \\
= & C_{2} \sum_{i=1}^{m-2} \alpha_{i} \cos \left(\beta_{i} \sqrt{\lambda_{n}}\right)-\frac{b}{4} \sum_{i=1}^{m-2} \alpha_{i} \cos \left(\beta_{i} \sqrt{\lambda_{n}}\right) \\
& -\frac{b \sqrt{\lambda_{n}}}{2} \sum_{i=1}^{m-2} \alpha_{i} \beta_{i} \sin \left(\beta_{i} \sqrt{\lambda_{n}}\right)
\end{aligned}
$$

From (3.15), (3.16) and $u(1)=\sum_{i=1}^{m-2} \alpha_{i} u\left(\beta_{i}\right)$, we have

$$
\sin \sqrt{\lambda_{n}}=\sum_{i=1}^{m-2} \alpha_{i} \beta_{i} \sin \left(\beta_{i} \sqrt{\lambda_{n}}\right)
$$

Applying the Schwarz inequality and (3.17), we have

$$
\begin{aligned}
1-\cos ^{2} \sqrt{\lambda_{n}} & =\sin ^{2} \sqrt{\lambda_{n}}=\left(\sum_{i=1}^{m-2} \alpha_{i} \beta_{i} \sin \left(\beta_{i} \sqrt{\lambda_{n}}\right)\right)^{2} \\
& \leq\left(\sum_{i=1}^{m-2} \beta_{i}^{2}\right)\left(\sum_{i=1}^{m-2} \alpha_{i}^{2} \sin ^{2}\left(\beta_{i} \sqrt{\lambda_{n}}\right)\right) \\
& =\left(\sum_{i=1}^{m-2} \beta_{i}^{2}\right)\left[\sum_{i=1}^{m-2} \alpha_{i}^{2}\left(1-\cos ^{2}\left(\beta_{i} \sqrt{\lambda_{n}}\right)\right]\right. \\
& =\left(\sum_{i=1}^{m-2} \beta_{i}^{2}\right)\left(\sum_{i=1}^{m-2} \alpha_{i}^{2}\right)-\left(\sum_{i=1}^{m-2} \beta_{i}^{2}\right)\left(\sum_{i=1}^{m-2} \alpha_{i}^{2} \cos ^{2}\left(\beta_{i} \sqrt{\lambda_{n}}\right)\right) .
\end{aligned}
$$


Combining $\cos \sqrt{\lambda_{n}}=\sum_{i=1}^{m-2} \alpha_{i} \cos \left(\beta_{i} \sqrt{\lambda_{n}}\right)$, we have

$$
\begin{aligned}
1 \leq & \cos ^{2} \sqrt{\lambda_{n}}+\left(\sum_{i=1}^{m-2} \beta_{i}^{2}\right)\left(\sum_{i=1}^{m-2} \alpha_{i}^{2}\right)-\left(\sum_{i=1}^{m-2} \beta_{i}^{2}\right)\left(\sum_{i=1}^{m-2} \alpha_{i}^{2} \cos ^{2}\left(\beta_{i} \sqrt{\lambda_{n}}\right)\right) \\
= & \left(\sum_{i=1}^{m-2} \alpha_{i} \cos \left(\beta_{i} \sqrt{\lambda_{n}}\right)\right)^{2}+\left(\sum_{i=1}^{m-2} \beta_{i}^{2}\right)\left(\sum_{i=1}^{m-2} \alpha_{i}^{2}\right)-\left(\sum_{i=1}^{m-2} \beta_{i}^{2}\right)\left(\sum_{i=1}^{m-2} \alpha_{i}^{2} \cos ^{2}\left(\beta_{i} \sqrt{\lambda_{n}}\right)\right) \\
= & \left(\sum_{i=1}^{m-2} \beta_{i}^{2}\right)\left(\sum_{i=1}^{m-2} \alpha_{i}^{2}\right)+\left[1-\left(\sum_{i=1}^{m-2} \beta_{i}^{2}\right)\right]\left(\sum_{i=1}^{m-2} \alpha_{i}^{2} \cos ^{2}\left(\beta_{i} \sqrt{\lambda_{n}}\right)\right) \\
& +\sum_{i \neq j} \alpha_{i} \alpha_{j} \cos \left(\beta_{i} \sqrt{\lambda_{n}}\right) \cos \left(\beta_{j} \sqrt{\lambda_{n}}\right) \\
\leq & \left(\sum_{i=1}^{m-2} \beta_{i}^{2}\right)\left(\sum_{i=1}^{m-2} \alpha_{i}^{2}\right)+\left[1-\left(\sum_{i=1}^{m-2} \beta_{i}^{2}\right)\right] \sum_{i=1}^{m-2} \alpha_{i}^{2}+\sum_{i \neq j} \alpha_{i} \alpha_{j} \\
= & \sum_{i=1}^{m-2} \alpha_{i}^{2}+\sum_{i \neq j} \alpha_{i} \alpha_{j}=\left(\sum_{i=1}^{m-2} \alpha_{i}\right)^{2} .
\end{aligned}
$$

Since $\sum_{i=1}^{m-2} \alpha_{i}<1$, we know that (3.18) is a contradiction. So (3.12) holds. By (3.10) and (3.11), we know that the algebraic multiplicity of characteristic value $\lambda_{n}$ is 1 .

Lemma 3.3 The linear operator $L$ is e(t)-continuous on $E$.

Proof Take $u_{0} \in E$. For any given $\epsilon>0$, we choose $\delta=\left(1-\sum_{i=1}^{m-2} \alpha_{i}\right) \epsilon$, when $\left\|u-u_{0}\right\| \leq \delta$, we have

$$
\begin{aligned}
\left|(L u)(t)-\left(L u_{0}\right)(t)\right| & \leq \int_{0}^{1} G(t, s)\left|u(t)-u_{0}(t)\right| d s \\
& \leq\left\|u-u_{0}\right\|\left(1+\frac{\sum_{i=1}^{m-2} \alpha_{i}}{1-\sum_{i=1}^{m-2} \alpha_{i}}\right)<\epsilon,
\end{aligned}
$$

where $e(t)=1$. Hence $L$ is $e(t)$-continuous on $u_{0} \in E$. By the arbitrariness of $u_{0}, L$ is $e(t)$ continuous on $E$.

Lemma 3.4 Suppose that $\left(\mathrm{H}_{2}\right)$ and $\left(\mathrm{H}_{3}\right)$ hold. Then $T$ is Fréchet differentiable at $\theta$ and $T_{\theta}^{\prime}=\eta L$.

Proof By $\left(\mathrm{H}_{3}\right)$, for any given $\epsilon>0$, there exists $\delta>0$, when $0<|x|<\delta$, we obtain

$$
\left|\frac{g(t, x)}{x}-\eta\right|<\epsilon, \quad \forall t \in[0,1] .
$$

Namely,

$$
|g(t, x)-\eta x|<\epsilon|x|, \quad \forall t \in[0,1], 0<|x|<\delta .
$$


So we have

$$
|g(t, u(t))-\eta u(t)|<\|G u-\eta u\| \leq \epsilon\|u\|, \quad \forall t \in[0,1],|u| \leq \delta .
$$

By $\left(\mathrm{H}_{2}\right)$, we have $T \theta=\theta$. By (3.19), we have

$$
\begin{aligned}
\|T u-T \theta-\eta L u\| & =\|(L G) u-(\eta L) u\|=\|L(G u-\eta u)\| \\
& \leq\|L\| \cdot\|G u-\eta u\| \leq \epsilon\|L\| \cdot\|u\|, \quad \forall\|u\| \leq \delta .
\end{aligned}
$$

So we have

$$
\lim _{\|u\| \rightarrow 0} \frac{\|T u-T \theta-\eta L u\|}{\|u\|}=0 .
$$

Namely, $T_{\theta}^{\prime}=\eta L$.

Lemma 3.5 Suppose that $\left(\mathrm{H}_{4}\right)$ holds. Then the Fréchet derivative $T_{\infty}^{\prime}=\gamma L$.

Proof By $\left(\mathrm{H}_{4}\right)$, for any given $\epsilon>0$, there exists $M>0$, when $|x| \geq M$, we have

$$
\left|\frac{g(t, x)}{x}-\gamma\right|<\epsilon, \quad \forall t \in[0,1] .
$$

Namely,

$$
|g(t, x)-\gamma x|<\epsilon|x|, \quad \forall t \in[0,1],|x| \geq M
$$

Let $\widetilde{M}=\max _{t \in[0,1],|x| \leq M}|g(t, x)-\gamma x|$. Then

$$
|g(t, x)-\gamma x| \leq \widetilde{M}+\epsilon|x|, \quad \forall t \in[0,1], x \in(-\infty,+\infty) .
$$

So

$$
\begin{aligned}
\|T u-\gamma L u\| & =\|(L G) u-(\gamma L) u\|=\|L(G u-\gamma u)\| \leq\|L\| \cdot\|G u-\gamma u\| \\
& \leq(\widetilde{M}+\epsilon\|u\|)\|L\|, \quad \forall u \in E .
\end{aligned}
$$

Therefore

$$
\lim _{\|u\| \rightarrow \infty} \frac{\|T u-\gamma L u\|}{\|u\|}=0 .
$$

Namely, $T_{\infty}^{\prime}=\gamma L$.

Theorem 3.1 Suppose that $\left(\mathrm{H}_{1}\right)-\left(\mathrm{H}_{5}\right)$ hold. Then BVP (1.1) has at least five nontrivial solutions: two positive solutions, one sign-changing solution, and two negative solutions.

Proof (i) By $\left(\mathrm{H}_{5}\right)$, we have

$$
g(t, \tau) \leq C \tau \quad \text { for } t \in[0,1] .
$$


So

$$
T(\tau)=\int_{0}^{1} G(t, s) g(s, \tau) d s \leq C \int_{0}^{1} G(t, s) \tau d s \leq C \tau\left(1+\frac{\sum_{i=1}^{m-2} \alpha_{i}}{1-\sum_{i=1}^{m-2} \alpha_{i}}\right) \leq \tau .
$$

Similarly, by $\left(\mathrm{H}_{5}\right)$, we have

$$
\begin{aligned}
T(-w) & =\int_{0}^{1} G(t, s) g(s,-w) d s \geq-C w \int_{0}^{1} G(t, s) d s \\
& \geq-C w\left(1+\frac{\sum_{i=1}^{m-2} \alpha_{i}}{1-\sum_{i=1}^{m-2} \alpha_{i}}\right) \geq-w .
\end{aligned}
$$

Take $\alpha=\min \{C \tau, C w\}$. Then, by (3.20) and (3.21), we have

$$
\alpha e(t)<\tau, \quad-w<-\alpha e(t)
$$

where $e(t)=1$.

From (3.20)-(3.22), condition (i) of Lemma 2.1 is satisfied.

(ii) By $\left(\mathrm{H}_{2}\right)$, we have $G(\theta)=\theta$. Similar to the proof of Lemma 3.4, $G$ is Fréchet differentiable at $\theta$. By Lemma 3.4, we know that $\frac{\lambda_{n}}{\eta}$ is the characteristic value of $T_{\theta}^{\prime}$. Since $\eta>\lambda_{1}$, $T_{\theta}^{\prime}$ has a characteristic value $\frac{\lambda_{1}}{\eta}<1$. Let $\psi(t)$ be a characteristic function corresponding to the characteristic value $\frac{\lambda_{1}}{\eta}<1$. Namely,

$$
\left(\frac{\lambda_{1}}{\eta} T_{\theta}^{\prime} \psi\right)(t)=\psi(t), \quad \forall t \in[0,1]
$$

By (3.2)-(3.4), we have

$$
(1-s) \frac{\sum_{i=1}^{m-2} \alpha_{i}}{1-\sum_{i=1}^{m-2} \alpha_{i}} \leq G(t, s) \leq 1+\frac{\sum_{i=1}^{m-2} \alpha_{i}(1-s)}{1-\sum_{i=1}^{m-2} \alpha_{i}} .
$$

So

$$
\lambda_{1} \frac{\sum_{i=1}^{m-2} \alpha_{i} \int_{0}^{1}(1-s) \psi(s) d s}{1-\sum_{i=1}^{m-2} \alpha_{i}} \cdot 1 \leq \psi(t) \leq \lambda_{1}\left[1+\frac{\sum_{i=1}^{m-2} \alpha_{i}}{2\left(1-\sum_{i=1}^{m-2} \alpha_{i}\right)}\right]\|\psi\| \cdot 1 .
$$

i.e.,

$$
\mu_{1} e(t) \leq \psi(t) \leq \mu_{2} e(t)
$$

where

$$
\mu_{1}=\lambda_{1} \frac{\sum_{i=1}^{m-2} \alpha_{i} \int_{0}^{1}(1-s) \psi(s) d s}{1-\sum_{i=1}^{m-2} \alpha_{i}}, \quad \mu_{2}=\lambda_{1}\left[1+\frac{\sum_{i=1}^{m-2} \alpha_{i}}{2\left(1-\sum_{i=1}^{m-2} \alpha_{i}\right)}\right]\|\psi\|, e(t)=1 .
$$

Then condition (ii) of Lemma 2.1 is satisfied.

(iii) From Lemma 3.5, $T_{\infty}^{\prime}=\gamma L$. So $T_{\infty}^{\prime}$ is increasing and $\frac{\lambda_{n}}{\gamma}$ is the characteristic value of $T_{\infty}^{\prime}$, where $\lambda_{n}$ is defined by $\left(\mathrm{H}_{1}\right)$. By $\left(\mathrm{H}_{4}\right)$, since $r\left(T_{\infty}^{\prime}\right)=\frac{\gamma}{\lambda_{1}}, \gamma>\lambda_{1}$, and $\gamma \neq \lambda_{n}$, we have 
that $r\left(T_{\infty}^{\prime}\right)>1$ and 1 is not a characteristic value of $T_{\infty}^{\prime}$. Hence condition (iii) of Lemma 2.1 holds.

From the above proof, Theorem 3.1 holds by Lemma 2.1.

\section{Application}

The following nonlinear four-point boundary value problem is studied:

$$
\left\{\begin{array}{l}
-u^{\prime \prime}(t)=g(t, u(t)), \quad 0 \leq t \leq 1, \\
u^{\prime}(0)=0, \quad u(1)=\frac{1}{4} u\left(\frac{1}{5}\right)+\frac{1}{2} u\left(\frac{1}{3}\right) .
\end{array}\right.
$$

From simple calculations, $\lambda_{1} \approx 0.5626, \lambda_{2} \approx 23.3709, \lambda_{3} \approx 70.0951$ are solutions of the following equation:

$$
\cos \sqrt{x}=\frac{1}{4} \cos \frac{\sqrt{x}}{5}+\frac{1}{2} \cos \frac{\sqrt{x}}{3} .
$$

Let

$$
g(t, x)= \begin{cases}2 x+\sqrt{x}-\frac{t}{64}-\frac{439}{64}, & t \in[0,1], x \in(4,+\infty), \\ (x-1)+\frac{9}{64}-\frac{t}{64}, & t \in[0,1], x \in(1,4), \\ \frac{9}{64}-\frac{t}{64}, & t \in[0,1], x \in\left(\frac{1}{8}, 1\right] \\ x+(1-t) x^{2}, & t \in[0,1], x \in\left[-1, \frac{1}{8}\right], \\ \frac{1}{72}(x+1)-t, & t \in[0,1], x \in(-27,-1), \\ 2 x+\sqrt[3]{x}+\frac{2039}{36}-t, & t \in[0,1], x \in(-\infty,-27] .\end{cases}
$$

From (4.2), we know that $g$ is continuous and increasing on $x ; g(t, 0)=0$.

$$
\begin{aligned}
& \liminf _{x \rightarrow 0} \frac{g(t, x)}{x}=1>\lambda_{1}, \\
& \limsup _{x \rightarrow \infty} \frac{g(t, x)}{x}=2>\lambda_{1} .
\end{aligned}
$$

Let $C=\frac{1}{5}, \tau=1, w=-27$, then

$$
\begin{aligned}
& \frac{g(t, \tau)}{\tau}=\frac{9}{64}-\frac{t}{64}<\frac{1}{5} \\
& \frac{g(t, w)}{w}=\frac{13}{972}+\frac{t}{27}<\frac{1}{5} .
\end{aligned}
$$

So, by Theorem 3.1, BVP (4.1) has at least two positive solutions, one sign-changing solution, and two negative solutions.

\section{Acknowledgements}

The authors wish to thank the reviewers for their valuable suggestions. 
Availability of data and materials

Data sharing not applicable to this article as no data sets were generated or analysed during the current study.

\section{Conflict of interests}

The authors declare that there is no conflict of interests regarding the publication of this article.

\section{Competing interests}

The authors declare that they have no competing interests.

Authors' contributions

All authors contributed equally to the writing of this paper. All authors read and approved the final manuscript.

\section{Publisher's Note}

Springer Nature remains neutral with regard to jurisdictional claims in published maps and institutional affiliations.

Received: 9 January 2020 Accepted: 24 March 2020 Published online: 05 June 2020

\section{References}

1. Timoshenko, S.: Theory of Elastic Stability. McGraw-Hill, New York (1961)

2. Gupta, C.P.: Solvability of a three-point nonlinear boundary value problem for a second order ordinary differential equations. J. Math. Anal. Appl. 168(2), 540-551 (1992)

3. Webb, J.R.L.: Positive solutions of some three point boundary value problems via fixed point index theory. Nonlinear Anal. 47, 4319-4332 (2001)

4. Xu, X.: Multiple sign-changing solutions for some m-point boundary value problems. Electron. J. Differ. Equ. 2004, 89 (2004)

5. Zhang, G.W., Sun, J.X.: Existence of positive solutions for singular second-order m-point boundary value problems Acta Math. Appl. Sin. Engl. Ser. 20, 655-664 (2004)

6. Zhang, G.W., Sun, J.X.: Multiple positive solutions of singular second order three-point boundary value problems. J. Math. Anal. Appl. 317, 442-447 (2006)

7. Rynne, B.P.: Second-order three-point boundary value problems with jumping nonlinearities. Nonlinear Anal. 68, 3294-3306 (2008)

8. Sun, J.X., Xu, X.A., O'Regan, D.: Nodal solutions for m-point boundary value problems using bifurcation methods. Nonlinear Anal. 68, 3034-3046 (2008)

9. Zhang, K.M., Xie, X.J.: Existence of sign-changing solutions for some asymptotically linear three-point boundary value problems. Nonlinear Anal. 70, 2796-2805 (2009)

10. Ma, R., An, Y: Global structure of positive solutions for superlinear second order $m$-point boundary value problems Topol. Methods Nonlinear Anal. 34, 279-290 (2009)

11. Webb, J.R.L., Infante, G.: Semi-positive nonlocal boundary value problems of arbitrary order. Commun. Pure Appl. Anal. 9, 563-581 (2010)

12. Bai, D.L., Feng, H.F: Eigenvalue for a singular second order three-point boundary value problem. J. Appl. Math. Comput. 38, 443-452 (2012)

13. Yu, C.D., Guo, Y.P., Ji, Y.D.: Existence of solutions for m-point boundary value problems on a half-line. Adv. Differ. Equ. 2009, Article ID 609143 (2009)

14. Cui, Y.J., Zou, Y.M.: Existence results and the monotone iterative technique for nonlinear fractional differential systems with coupled four-point boundary value problems. Abstr. Appl. Anal. 2014, Article ID 242591 (2014)

15. Li, H.Y.: Existence of nontrivial solutions for superlinear three-point boundary value problems. Acta Math. Appl. Sin. Engl. Ser. 33, 1043-1052 (2017)

16. Wei, Y.F., Bai, Z.B., Sun, S.J.: On positive solutions for some second-order three-point boundary value problems with convection term. J. Inequal. Appl. 2019, Article ID 72 (2019)

17. Li, H.Y., Zhang, J.T.: Global structure of positive solutions for some second order multi-point boundary value problems. J. Funct. Spaces 2017, Article ID 1014250 (2017)

18. Li, H.Y., Zhang, J.T.: Existence of nontrivial solutions for some second-order multipoint boundary value problems. J. Funct. Spaces 2018, Article ID 6486135 (2018)

19. Sang, Y.B., Meng, Q., Zhu, Z.Y.: Sign-changing solutions for asymptotically linear operator equations and applications. Bound. Value Probl. 2015, Article ID 184 (2015)

20. Li, F.Y., Liang, Z.P., Zhang, Q., Li, Y.H.: On sign-changing solutions for nonlinear operator equations. J. Math. Anal. Appl. $327,1010-1028(2007)$

21. Guo, D.J.: Nonlinear Functional Analysis, 2nd edn. Shandong Sci. and Tech. Press, Jinan (2001)

22. Guo, D.J., Lakshmikantham, V.: Nonlinear Problems in Abstract Cones. Academic Press, San Diego (1988)

23. Sun, J.X.: Nonlinear Functional Analysis and Applications. Science Press, Beijing (2008) 